\title{
Tricuspid Valve Fungal Endocarditis in a Patient with Breast Cancer and an Implantable Chemotherapy Venous Access Port
}

\section{Petros Fountoulakis ${ }^{1}$, Aikaterini Siama ${ }^{1}$, Aimilianos Kalogeris ${ }^{1}$, Marianthi lliopoulou², Athanasios Tsoukas ${ }^{1}$, Athanasios Manolis ${ }^{1}$}

1. Cardiac Echocardiography Department, Asklepeion Voulas General Hospital, Voula, Athens, Greece

2. 7th Respiratory Medicine Department and Asthma Center, Athens Chest Hospital "Sotiria", Athens, Greece

\section{Corresponding author:}

Vas. Pavlou Ave. 1 Voula 16673

Athens Greece

Tel: 00302132163221

E-mail: pfountou@yahoo.gr

\section{Highlights}

Fungal endocarditis, a rare and lethal infection, is aetiologically connected with Candida and Aspergillus species. Among these two agents, Candida is a common nosocomial infection with increasing rates over the last years and mortality up to $40 \%$ in cases of systemic candidiasis. In the present paper, we describe the case of a 58 year old woman with metastatic breast cancer under palliative chemotherapy who was hospitalized for recurrent episodes of fever due to fungal endocarditis of the implantable venous access port. Such cases may elude the attention of the physician and need to be taken into account especially in oncologic patients with implantable devices under chemotherapy regimens. The treatment of Candida endocarditis can be difficult because of the formation of biofilms on prosthetic devices. The prognosis of these patients may be ameliorated with the combination of fungal and invasive treatment.

Keywords: Cardiooncology; right sided infective endocarditis; fungal endocarditis; intraport catheter; breast cancer

Citation: $\quad$ Fountoulakis P, Siama A, Kalogeris A, lliopoulou M, Tsoukas A, Manolis A. Tricuspid Valve Fungal Endocarditis in a Patient with Breast Cancer and an Implantable Chemotherapy Venous Access Port. International Cardiovascular Forum Journal. 2019;16:40-41. DOI: 10.17987/icfj.v16i0.581

Right sided infective endocarditis (IE) accounts for less than $10 \%$ of all IE cases. Predisposing factors include portal of entry, implanted foreign material and unrepaired congenital heart disease with conduit. [1]

Fungal endocarditis (FE) constitutes the most severe form of IE and is aetiologically connected predominantly to Candida and Aspergillus species. Among these two agents, Candida species is a common nosocomial infection with increasing prevalence and mortality rates up to $40 \%$ in cases of systemic candidiasis. [2] Individuals with different forms of solid or hematological malignancies, under chemotherapy regimens or bone marrow transplantation comprise a particularly susceptible patient population. [3]

A 58 year old woman with personal history of triple negative breast adenocarcinoma stage IV under palliative chemotherapy, administered for metastatic mass shrinkage in the gastrointestinal tract, was admitted to the Emergency Department of our Hospital due to persistent fever, malaise and dyspnoea on effort (MMRC 2-3). Chemotherapy was infused via an implantable venous access port (intraport catheter). Her heart auscultation revealed a holosystolic ejection type murmur of $3 / 6$ located in the third intercostal space of changing quality. Candida tropicalis was isolated in three separate blood cultures.

Transthoracic echocardiography demonstrated a good overall left ventricular systolic function. The right cavities were moderately dilated with moderate tricuspid regurgitation and a pulmonary pressure estimated at $45 \mathrm{mmHg}$. A large vegetation (approximately $2 \mathrm{~cm}$ maximal diameter) at the atrial surface of the posterior and diaphragmatic leaflets of the tricuspid valve with parts of the vegetation periodically apparent in the right ventricle was observed.

Trans-oesophageal echocardiography confirmed the findings of the transthoracic study and elucidated in the bicaval view the connection of the vegetation in the tricuspid valve with the edge of the intraport catheter (Figure 1).

The computed tomography scan revealed pulmonary embolism in the segmental branches of the bronchial tree and a circumscribed peripheral pulmonary infarct of the left inferior lobe (Figure 2).

A multidisciplinary team concluded that the best treatment 

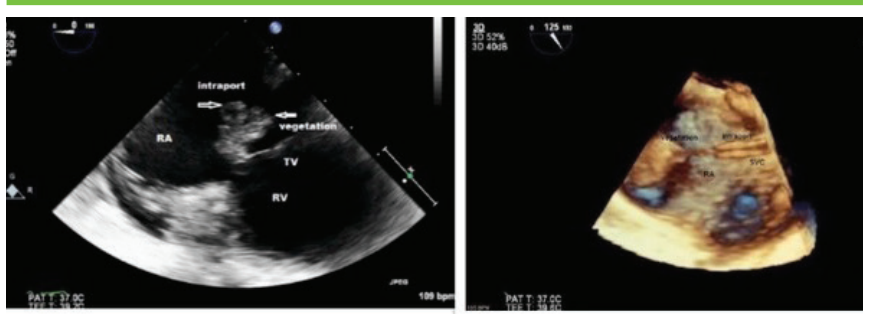

Figure 1.

strategy would require aggressive intravenous combined antifungal therapy until eradication followed by removal of the implantable venous access port. The invasive approach was preferred despite the risk of vegetation dissemination and further embolism since preservation of the intraport was considered detrimental for the patient. Intraport removal was uncomplicated and the patient was discharged in an improved state.

It is noteworthy that the culture of the end of this device was negative for fungal or other pathogen. Thus, an additional likely mechanism of $F E$ in our patient could be the translocation of fungi from the site of the intestinal occlusion due to immunosuppression caused by the chemotherapy, prolonged antimicrobial use and parenteral nutrition. The patient was initially treated with intravenous administration of micafungin for six weeks and then per os fluconazole as chemoprophylaxis.

Numerous studies have shown the increasing rates of implantable access port infections in oncologic patients and their elevated mortality rates. [4] The subset of patients with prosthetic devices represents a population with increased vulnerability to FE as demonstrated by the multicenter cohort study of Fernadez-Cruz et al. [5]

Candida endocarditis has the specific ability to create biofilms on prosthetic devices leading to the reduction of the effect of antifungal agents. As a result combination treatment with echinocandins and azoles can lead to important benefits. [6] The decision of the appropriate antimicrobial agent is difficult as it is necessary to balance the benefits and side effects of this treatment as well as take into account possible drug interactions. $[7,8]$ Septic pulmonary embolism with multiple loci is a frequent complication in right sided infective endocarditis. Furthermore, surgical removal of the prosthetic device if feasible in addition to antifungal treatment is linked to a more favorable prognosis. [9]

In our case, the prolonged antibiotic therapy due to recurrent bronchial pneumonia and ileus and was also an important risk factor for systemic candidiasis due to disruption of the intestinal microflora and subsequent colonization by Candida species. Thus a positive blood culture for Candida spp. in the setting of protracted antibiotic regimens should never be overlooked. Currently the rates of candidemia have significantly increased because of the rising number of patients being at risk. The great majority of fungal endocarditis episodes represented healthcareassociated infections (94\%), caused most frequently by Candida species (mainly non-albicans Candida), with high rates of mortality and recurrence. These findings are in line with previously published observational studies on fungal endocarditis. [10]
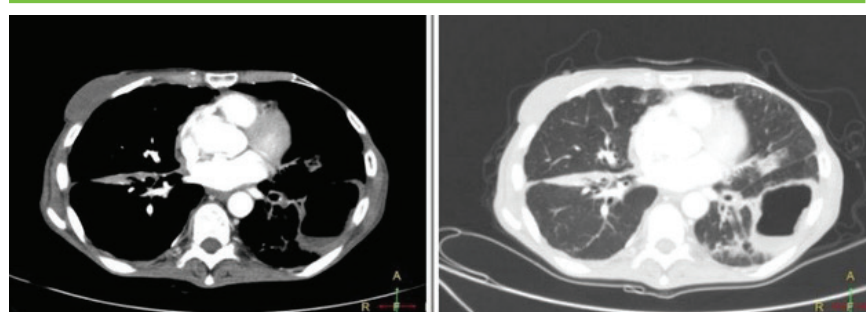

Figure 2.

possibility of fungal endocarditis should always be considered. Consequently, the physicians should be alert for early detection and initiation of treatment since the prognosis of these patients may be ameliorated.

\section{Declarations of Interest}

The authors declare no conflicts of interest.

\section{Acknowledgements}

The authors state that they abide by the "Requirements for Ethical Publishing in Biomedical Journals". [11]

\section{References}

1. Yuan S-M. Right-sided infective endocarditis: recent epidemiologic changes. Int J Clin Exp Med. 2014 Jan 15;7(1):199-218.

2. Yuan S-M. Fungal Endocarditis. Braz J Cardiovasc Surg. 2016 Jun;31(3):252-255. doi:10.5935/1678-9741.20160026.

3. Simon MS, Somersan S, Singh HK, Hartman B, Wickes BL, Jenkins SG, et al. Endocarditis caused by Rhodotorula infection. J Clin Microbiol. 2014 Jan;52(1):374-378. doi:10.1128/JCM.01950-13.

4. Lebeaux D, Larroque B, Gellen-Dautremer J, Leflon-Guibout V, Dreyer $\mathrm{C}$, Bialek S, et al. Clinical outcome after a totally implantable venous access port-related infection in cancer patients: a prospective study and review of the literature. Medicine. 2012 Nov;91(6):309-318. doi:10.1097/ MD.0b013e318275ffe1.

5. Fernández-Cruz A, Muñoz P, Sandoval C, Fariñas C, Gutiérrez-Cuadra $\mathrm{M}$, Pericás Pulido JM, et al. Infective endocarditis in patients with cancer: a consequence of invasive procedures or a harbinger of neoplasm? A prospective, multicenter cohort. Medicine. 2017 Sep;96(38):e7913. doi:10.1097/MD.0000000000007913.

6. Pappas PG, Kauffman CA, Andes D, Benjamin DK, Calandra TF, Edwards $\mathrm{JE}$, et al. Clinical practice guidelines for the management of candidiasis: 2009 update by the Infectious Diseases Society of America. Clin Infect Dis. 2009 Mar 1;48(5):503-535. doi:10.1086/596757.

7. Lejko Zupanc T, Logar M. Candida endocarditis: A review of twelve episodes in eleven patients. J Heart Valve Dis. 2017 Jan;26(1):98-102.

8. Reyes HA, Carbajal WH, Valdez LM, Lozada C. Successful medical treatment of infective endocarditis caused by Candida parapsilosis in an immunocompromised patient. BMJ Case Rep. 2015 Oct 9;2015. doi:10.1136/bcr-2015-212128.

9. Koumallos N, Paschalis A, Antoniades C, Tousoulis D, Simpsiris P, Tolios I, et al. Valve replacement for Brucella endocarditis: two case reports. Int $J$ Cardiol. 2008 Jul 4;127(2):e83-5. doi:10.1016/j.ijcard.2007.04.081.

10. Arnold CJ, Johnson M, Bayer AS, Bradley S, Giannitsioti E, Miró JM, et al. Candida infective endocarditis: an observational cohort study with a focus on therapy. Antimicrob Agents Chemother. 2015 Apr;59(4):2365-2373. doi:10.1128/AAC.04867-14.

11. Shewan LG, Coats AJS, Henein MY. Authors' Responsibilities and Ethical Publishing. International Cardiovascular Forum Journal 2018;13:3-4, doi: 10.17987/icfj.v13i0.525 